\title{
Survey Evaluating Sleep Education Catalyzed Change in Residency Training
}

\author{
Demetra S. Stamm, Sandra Taylor, Uyen Thao Nguyen, Kimberly Hardin* \\ University of California, Davis, USA \\ Email: ${ }^{*}$ drkahardin@gmail.com
}

Received 13 May 2015; accepted 30 June 2015; published 3 July 2015

Copyright (C) 2015 by authors and Scientific Research Publishing Inc.

This work is licensed under the Creative Commons Attribution International License (CC BY). http://creativecommons.org/licenses/by/4.0/

\section{Abstract}

Background: Despite the high prevalence and negative effects of sleep disorders, sleep issues often remain unexplored during medical encounters. Research has shown that primary care physicians regard their knowledge as inadequate. Objective: We investigated residents' perceived adequacy of sleep education and level of competency in diagnosing and treating sleep disorders. Methods: A questionnaire via Survey Monkey was administered to senior residents in University of California, Davis (UCD) from family medicine, internal medicine, neurology, and psychiatry to assess perception of their knowledge and training of sleep disorders. Likert scale ratings were used, from 1 (not adequate/competent) to 5 (extremely adequate/competent). Non-parametric statistical methods were used to evaluate differences in survey responses among specialties and assess the correlation between survey responses. Results: Only 33 residents responded with a $29.5 \%$ response rate. Neurology residents routinely rotate with a sleep medicine attending and subsequently reported the highest self-competency, adequacy of training, hours of didactics received and frequency of asking patients about sleep. All other residents reported receiving insufficient sleep medicine education. The combined mean score was 1.5 across the specialties regarding adequacy of sleep education in their respective medical schools with $31.3 \%$ reporting no sleep medicine training. Conclusions: This study demonstrated that there is a perceived deficit in most residents' training and competency regarding sleep disorders. These results prompted 2 of the 4 residency programs to change their educational structure with electives in clinical sleep medicine. We believe that this paper illuminates potential need for increasing sleep medicine education throughout various levels of training. Nationwide educational research is needed to promote ACGME to incorporate the fundamentals of sleep medicine into core curriculum. Current Knowledge/Study Rationale: There are no studies, to our knowledge, directly measuring residents' perceived adequacy of sleep medicine education provided and their perceived level of competency in diagnosing and treating sleep disorders. The aim of this study was to evaluate both the amount of time and quality of sleep education provided to residents as insufficient knowledge in addressing sleep problems may reflect gaps in educational requirements and standards within residency training. Study Impact: This

\footnotetext{
"Corresponding author.

How to cite this paper: Stamm, D.S., Taylor, S., Nguyen, U.T. and Hardin, K. (2015) Survey Evaluating Sleep Education Catalyzed Change in Residency Training. International Journal of Clinical Medicine, 6, 444-450. 
study revealed a perceived deficit in most residents' training and competency regarding sleep disorders. This study stimulated half of the UC Davis residency programs evaluated to offer electives in clinical sleep medicine suggesting that the study highlighted need for additional educational opportunities in sleep medicine.

\section{Keywords}

\section{Survey Evaluating, Sleep Education, Residency Training}

\section{Introduction}

Over 50 million Americans suffer from some sleep-related problems that can result in chronic sleep deprivation [1]. Obstructive sleep apnea has been linked with cardiovascular disease, diabetes, mental health issues, and cognitive dysfunction that can result in fatal accidents [1]-[3]. Despite the high prevalence and negative effects of sleep disorders, sleep issues often remain unexplored during medical encounters. In 2000, Papp et al. [4] surveyed 580 primary care physicians with $90 \%$ rating their knowledge of sleep medicine as fair or poor.

Inadequacies addressing sleep problems in patients may reflect gaps in educational requirements and standards within residency training. Studies have evaluated educators' views on sleep medicine education in medical school or residency. A 1998 nationwide survey of US medical schools found that only 2.1 hours were devoted to teaching topics in sleep medicine [5]. A 1993 survey of US medical schools showed that 37 schools reported no structured teaching time [6]. More than two-thirds of respondents felt that the sleep education was inadequate. The main obstacles included unavailability of qualified faculty, inadequate curriculum time and need for additional educational resources [6].

Three nationwide studies have been published that surveyed program directors' opinion on residents' training in sleep medicine [7]-[9]. A study in 2002, surveyed 177 USA psychiatry program directors with a 66\% response rate, revealing that most programs (82\%) offered didactics in sleep [8]. Only $44 \%$ offered a sleep elective and 10 programs had discontinued sleep electives [8]. The authors conclude that an overall "decrease in educational opportunities may result in fewer newly trained psychiatrists entering the sleep field" [8]. A study in 2010 sent sleep surveys to 103 otolaryngology residency program directors with a $46 \%$ response [9]. Forty-four percent rated their satisfaction with residents' education in sleep medicine as "fair", indicating program directors' perceived need for improvement in sleep education delivered [9]. Another study in 2010 surveyed 126 neurology residency program directors with a $46 \%$ response rate. This study revealed that $81 \%$ of neurology programs offer a sleep rotation and that programs that have more "sleep medicine teaching resources were more likely to report resident entering a sleep medicine training program” [10].

The aim of this study was to investigate both the amount of time and quality of sleep education provided to residents. Specifically, we evaluated trainees' beliefs on how sufficient their sleep medicine education was and their perceived competence in diagnosing and treating sleep disorders.

\section{Methods}

\subsection{Subjects}

A prospective survey evaluating residents quality of sleep medicine education was conducted from 2012-2013. The study was approved by the institutional review board at UCD. Participants were senior residents from the departments of family medicine (FM), internal medicine (IM), neurology (Neuro), and psychiatry (Psych) at the UCD. These residency specialties were selected because of increased likelihood of having patients present with sleep problems. To gather the residents complete training experience, the survey evaluated both their medical school and residency training experience.

\subsection{Survey Design}

A survey was created with 16 multiple choice questions for residents (11 for residency training and 5 for medical school) using an online survey platform (Survey Monkey, Palo Alto, CA, www.surveymonkey.com). The 
survey was sent to residency coordinators who forwarded to senior residents. This is a novel study therefore there was no available validated survey tool. Suggestions on survey design from a JGME editorial by Magee et al. [10] were used.

Four areas in sleep medicine education were evaluated: 1) hours of didactic instruction devoted to sleep medicine; 2) frequency of residents inquiring about sleep and providing sleep education to patients; 3 ) residents perceived self-competency in treating sleep disorders; 4) residents perceived adequacy of clinical training in sleep medicine.

Box 1.

Resident survey questions presented in this paper:

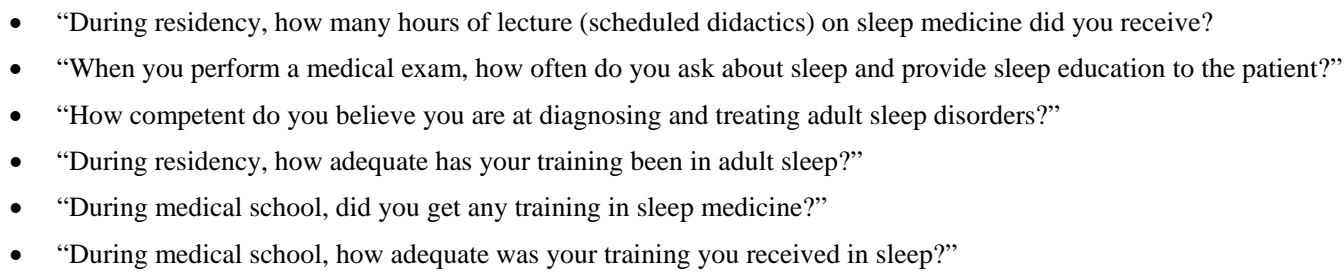

SUPPLEMENTAL 1 details full questionnaire.

Hours of training were converted to numerical scores as follows: None $=0$, Less than $2=1,2-5$ hours $=3.5$, More than 5 hours $=6$. Residents report of frequency of asking about sleep categories were converted to a 5 point scale: Never $=1$, Rarely $=2$, Sometimes $=3$, Very often $=4$ and Always or Almost Always $=5$. Assessing residents' perceived competency and adequacy of training were recorded using a 5-point Likert scale $(1=$ not competent/adequate; 5 = extremely competent/adequate). Question regarding whether resident had training in medical school had answer options of “yes”, "no” and "I don’t know”.

\subsection{Statistical Analysis}

Likert scale questions were summarized in means. Kruskal-Wallis tests, the non-parametric alternative to the ANOVA, were used to test for differences in median scores (e.g., self-reported adequacy of training) among the specialities. For significant $(\mathrm{p}<0.05)$ Kruskal-Wallis tests, Wilcoxon tests were used to conduct pairwise comparisons of specialities with a Bonferroni adjustment to maintain the family-wise error at 0.05 . Spearman's rank correlation was used to evaluate correlations among self-reported adequacy, competency, hours of didactic training and the frequency at which residents asked adults about sleep.

\section{Results}

\subsection{Response Rate}

A total of 112 residents were sent the survey over two years. Figure 1 shows combined responses rates from each specialty, demonstrating 33 residents responded with a $29.5 \%$ response rate. The response rate was 50\% (5 of 10 ) and $12.5 \%$ (2 of 16) from neurology and psychiatry residents, respectively. Because only 2 psychiatry residents responded, psychiatry was excluded from further statistical analysis.

\subsection{Didactic Hours}

Hours of didactic lecture differed significantly among the residency programs $(p=0.002)$. Pairwise comparisons showed that neurology residents received more didactic time than FM $(p=0.006)$ and IM $(p=0.005)$. The combined data showed that over $49 \%$ of residents reported "less than 2 hours" of didactic hours devoted to sleep medicine education. Only 9.8\% of residents rated that they had "more than 5 hours" (Figure 2). When evaluating programs separately, the majority of family medicine and internal medicine responded with "less than 2 hours", whereas neurology residents reported "more than 5 hours".

\subsection{Frequency of Asking Patients about Sleep}

Median frequency of asking adults about sleep differed significantly among the 3 specialties (Kruskal-Wallis $\mathrm{p}=$ 


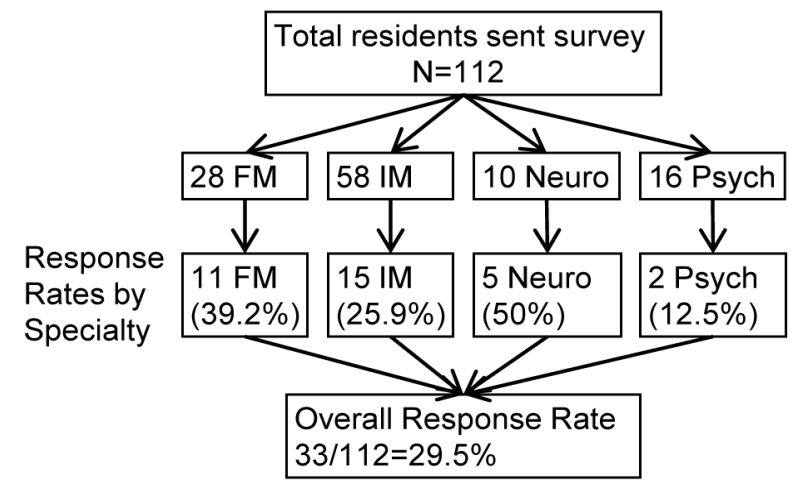

Figure 1. Response rates by specialty.

\title{
Hours of Lecture in Sleep Medicine During Residency
}

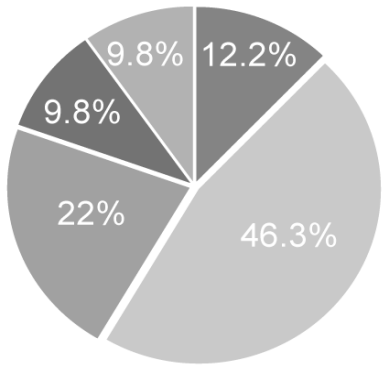

\author{
None \\ Less than 2 hours \\ 2-5 hours \\ More than 5 hours \\ Unknown
}

Figure 2. Results for survey question: "Hours of didactic teaching”.

0.011). Pairwise comparisons showed neurology residents to ask about sleep significantly more frequently than internal medicine $(\mathrm{p}=0.012)$ and family medicine $(\mathrm{p}=0.049)$. The majority of family medicine residents' reported "sometimes" (36\%, 4/11) and "rarely" (36\%, 4/11). The majority of internal medicine residents reported "sometimes" (64\%, 9/14), whereas the two psychiatry residents reported "very often” (100\%; 2 of 2). Notably, the majority of the neurology residents responded always/almost always (60\%; 3 of 5). The means for the combined data was 3.1 correlating to asking patients "sometimes" about sleep. In contrast, neurology residents had highest mean of 4.4 correlating to "very often" to "always".

One of the survey questions was the following: "If you infrequently ask about sleep what is the main reason." The majority of residents responded with the option: "Does not appear relevant to patient’s chief complaint.”

\subsection{Residents Perceived Self Competency}

For the competency question a total of 32 residents responding with a mean score of 2.9. Neurology had highest mean of 3.6, whereas the other specialties (IM, FM, Psych) ranged from 2.5 to 2.8 as shown in Table 1 . Although there was a difference in the mean for self-reported competency among the 3 specialties, it did not reach statistical significance $(p=0.059)$.

\subsection{Residents' Report of Adequacy of Resident Training in Sleep Medicine}

Self-reported adequacy of training differed significantly among the 3 specialties (FM, IM, Neuro) $(p=0.004)$. Pairwise comparisons with a Bonferroni adjustment revealed that neurology residents rated the adequacy of their training higher than the other specialties (FM, p $=0.013$ and IM, p = 0.007). The adequacy question, had similar results to the competency question, with a total of 32 residents responding and a mean of 2.6. Notably, the highest rated adequacy of training was from neurology residents with mean $=4.6$; psychiatry residents had the lowest reported training adequacy with a mean of 2 (Table 2). 
Table 1. Results for survey question: "How competent do you believe you are at diagnosing and treating adult sleep disorders” in a 5-point Likert scale.

\begin{tabular}{cccc}
\hline Specialty & $\mathrm{N}$ & $\begin{array}{c}\text { Mean } \\
\text { Median })\end{array}$ & $\begin{array}{c}\text { Standard Deviation } \\
\left(25^{\text {th }}, 75^{\text {th }} \text { Quartiles }\right)\end{array}$ \\
\hline Family Medicine & 11 & 2.7 & 0.8 \\
& & $(3)$ & $(2,3)$ \\
Internal Medicine & 14 & 2.8 & 0.6 \\
& & $(3)$ & $(2.25,3)$ \\
Neurology & 5 & $(4)$ & 0.5 \\
& & 2.5 & $(3,4)$ \\
Psychiatry & 2 & $(2.5)$ & 0.7 \\
& & 2.9 & $(2.25,2.75)$ \\
Total & 32 & $(3)$ & 0.7 \\
\end{tabular}

$\mathrm{N}=$ number of respondents. The 5-point Likert response options ranged from 1 = not competent to 5 = extremely competent.

Table 2. Results for survey question: "How adequate has your training been in adult sleep?” with responses in a 5-point Likert scale.

\begin{tabular}{cccc}
\hline Specialty & $\mathrm{N}$ & $\begin{array}{c}\text { Mean } \\
(\text { Median })\end{array}$ & $\begin{array}{c}\text { Standard Deviation } \\
\left(25^{\text {th }}, 75^{\text {th }} \text { Quartiles }\right)\end{array}$ \\
\hline Family Medicine & 11 & 2.3 & 0.9 \\
& & $(2)$ & 0.8 \\
Internal Medicine & 14 & 2.2 & $(2,3)$ \\
& & $(2)$ & 0.9 \\
Neurology & 5 & 4.6 & $(5,5)$ \\
& & 2 & 0 \\
Psychiatry & 2 & $(2)$ & $(2,2)$ \\
& & 2.6 & 0.7 \\
Total & 32 & $(2)$ & $(2,3)$ \\
\hline
\end{tabular}

$\mathrm{N}=$ number of respondents. The 5 -point Likert response options ranged from $1=$ not adequate to $5=$ extremely adequate.

\subsection{Correlations between Residents' Responses}

Combining the residents responses showed a higher reported competency with a mean $=2.6$ compared to adequacy of training a mean $=2.3$, correlating to a "somewhat" rating. There were significant correlations between adequacy and competency $(p=0.0006$, Spearman's rho $=0.606)$; hours of didactics and perceived competency $(p=0.018$, Spearman's rho $=0.444)$ and between adequacy and hours of lecture $(p<0.0001$, Spearman's rho $=$ 0.682). The frequency at which residents asked adult patients about sleep was significantly positively correlated with self-reported adequacy $(p=0.008$, Spearman's rho $=0.457)$ and competency $(p=0.002$, Spearman's rho $=$ 0.522 ).

\subsection{Medical School: Sleep Medicine Education}

Residents were also asked whether they received sleep medicine education during medical school, $53.1 \%$ of residents responded "yes", 31.3\% as "no", and 15.6\% as "I don't know". The combined mean across the specialties for how adequate sleep medicine education during medical school was 1.5 (STD \pm 0.83 ) indicating most residents felt their medical school sleep education was inadequate.

\section{Discussion}

This is the first study, to our knowledge, that evaluates how residents' perceive the adequacy of their education in principles of sleep medicine and their perceived level of competency in diagnosing and treating sleep disorders. This study illustrates that a gap exists in educational training and a lack of competency in sleep medicine by residents who will be likely to encounter patients with sleep issues. Given the high prevalence and negative effects of sleep disorders, education of our trainees is essential to provide care for sleep issues that have often re- 
mained unexplored during medical encounters.

We wish to address limitations to this study early in our discussion so that results may be interpreted in context. Firstly, the low response rate of residents may introduce nonresponse bias [11]. Thus the response of one individual can have a dramatic impact on the analysis and, in particular, the outcome by specific residency program. The low response rate may have been due to time constraints and timing during the academic year (the last quarter) that participation was requested, and/or non-mandatory participation. Moreover, the low response rate may reflect lack of emphasis placed on sleep medicine education training leading to less interest in participating. Secondly, survey based studies may introduce recall bias. Thirdly, the study results reflect residents' interpretation of the survey and personal perceptions, which may also introduce interpretation bias. Lastly, since the study was conducted only at one institution, there is limited generalizablity.

Despite these limitations, there were important trends identified that would seem to be due to more that "chance" occurrence. The majority of residents reported receiving less than 2 hours of didactics in sleep medicine topics and marginal adequacy in sleep education during medical school and residency. Residents reported variability in medical school training experiences with 31.3\% indicating "no" experience. Evaluation of the individual programs revealed that neurology residents had the greatest questionnaire response rate, reported the greatest level of perceived self-competency, adequacy of training, and highest number of didactic hours and greatest frequency of asking patients about sleep. We further looked at the structure of the neurology program's curriculum and found that all neurology residents attend a clinic supervised by a sleep boarded neurologist. Therefore, increasing residents' exposure to sleep medicine didactics and supervision with a sleep medicine specialist likely impacted the residents' ability to address sleep issues and improved residents' competency in this area.

The field of sleep medicine may be underappreciated as an integral role of physical and psychological health by both the individual resident and the training institution. There are no current ACGME training requirements regarding resident knowledge of sleep disorders for the programs evaluated in this study; only neurology mentions requirements for faculty to have knowledge of sleep disorders [12]. A white paper evaluating the structure of sleep medicine programs at academic institutions, reflected that there is insufficient structured curriculum, insufficient faculty trained in sleep medicine and administrative support at most academic institutions to foster the importance and development of sleep medicine [13] [14].

Despite the low response rate and the low sample size, this study seemed to stimulate a change in residency education at this institution. The positive educational outcome involved creation of sleep medicine elective opportunities in two departments-internal medicine and psychiatry. Residents from these departments have now actively participated in sleep clinics and didactics in sleep medicine have been instituted in their core lecture content. It is unclear if these curriculum changes were driven by the residents and/or program directors. Either way, this study suggests that knowledge of this survey may have highlighted deficits in training and promoted additional educational opportunities.

\section{Conclusion}

Overall, this study strongly supports that most senior level residents feel that a small amount of time is devoted to sleep medicine didactics during residency and that resident perception of the adequacy of training and their competency in sleep evaluation is low. Successful integration of sleep medicine knowledge and application will likely require exposure to sleep medicine throughout medical training [15]. Further research is needed to delineate specific training deficits. The American Academy of Sleep Medicine's Curriculum Development Advisory Committee has recently initiated a survey of sleep medicine education in US medical schools. We are hopeful that these types of research efforts lead to national recognition demonstrating the need for more emphasis on sleep medicine education during both medical school and residency training.

\section{Acknowledgements}

The authors are grateful for faculty discretionary funds provided by the UC Davis Department of Internal Medicine, Division of Pulmonary, Critical Care and Sleep Medicine which allowed this research to be possible. The authors thank the UCD departments of family medicine, internal medicine, psychiatry and neurology for their participation in the study. The authors wish to also thank Julie Rainwater, $\mathrm{PhD}$ for her assistance and guidance with survey design. 


\section{Funding}

Kimberly Hardin, MD used faculty discretionary funds from UC Davis Department of Internal Medicine, Division of Pulmonary, Critical Care and Sleep Medicine.

\section{References}

[1] National Institute of Health Sleep Disorders Research Plan (2011). http://www.nhlbi.nih.gov/files/docs/resources/sleep/201101011NationalSleepDisordersResearchPlanDHHSPublication 11-7820.pdf

[2] Morgenstern, M., Wang, J., Beatty, N., Batemarco, T., Sica, A.L. and Greenberg, H. (2014) Obstructive Sleep Apnea: An Unexpected Cause of Insulin Resistance and Diabetes. Endocrinology Metabolism Clinics of North America, 43, 187- 204. http://dx.doi.org/10.1016/j.ecl.2013.09.002

[3] Gupta, M.A. and Knapp, K. (2014) Cardiovascular and Psychiatric Morbidity in Obstructive Sleep Apnea (OSA) with Insomnia (Sleep Apnea plus) versus Obstructive Sleep Apnea without Insomnia: A Case-Control Study from a Nationally Representative US Sample. PLoS One, 9, e90021. http://dx.doi.org/10.1371/journal.pone.0090021

[4] Papp, K.K., Penrod, C.E. and Strohl, K.P. (2002) Knowledge and Attitudes of Primary Care Physicians towards Sleep and Sleep Disorders. Sleep and Breathing, 6, 103-109. http://dx.doi.org/10.1055/s-2002-34317

[5] Rosen, R., Mahowald, M., Chesson, A., et al. (1998) The Taskforce 2000 Survey on Medical Education in Sleep and Sleep Disorders. Sleep, 21, 235-238.

[6] Rosen, R.C., Rosekind, M., Rosevear, C., Cole, W.E. and Dement, W.C. (1993) Physician Education in Sleep and Sleep Disorders: A National Survey of U.S. Medical Schools. Sleep, 16, 249-254.

[7] Krahn, L.E., Hansen, M.R. and Tinsley, J.A. (2002) Psychiatric Residents’ Exposure to the Field of Sleep Medicine: A Survey of Program Directors. Academic Psychiatry, 26, 253-256. http://dx.doi.org/10.1176/appi.ap.26.4.253

[8] Shen, T., Shimahara, E., Cheng, J. and Capasso, R. (2011) Sleep Medicine Clinical and Surgical Training during Otolaryngology Residency: A National Survey of Otolaryngology Residency Programs. Otolaryngology-Head and Neck Surgery, 145, 1043-1048. http://dx.doi.org/10.1177/0194599811416765

[9] Avidan, A.Y., Vaughn, B.V. and Silber, M.H. (2015) The Current State of Sleep Medicine Education in US Neurology Residency Training Programs: Where Do We Go From Here? Journal of Clinical Sleep Medicine, 9, 281-286. http://dx.doi.org/10.5664/jcsm.2502

[10] Magee, C., Rickards, G., Byars, L. and Artino, A.R. (2013) Tracing the Steps of Survey Design: A Graduate Medical Education Research Example. Journal of Graduate Medical Education, 5, 1-5. http://dx.doi.org/10.4300/JGME-D-12-00364.1

[11] Johnson, T.P. and Wislar, J.S. (2012) Response Rates and Nonresponse Errors in Surveys. JAMA, 307, 1805-1806. http://dx.doi.org/10.1001/jama.2012.3532

[12] ACGME Program Requirements for GME in Neurology (2014). https://www.acgme.org/acgmeweb/Portals/0/PFAssets/ProgramRequirements/180_neurology_07012014.pdf

[13] Chervin, R.D., Chesson, A.L., Benca, R.M., et al. (2013) Organization and Structure for Sleep Medicine Programs at Academic Institutions: Part 1-Current Challenges. Sleep, 36, 795-801. http://dx.doi.org/10.5665/sleep.2690

[14] Chesson, A.L., Chervin, R.D., Benca, R.M., et al. (2013) Organization and Structure for Sleep Medicine Programs at Academic Institutions: Part 2-Goals and Strategies to Optimize Patient Care, Education, and Discovery. Sleep, 36, 803-811. http://dx.doi.org/10.5665/sleep.2692

[15] Strohl, K.P. (2011) Sleep Medicine Training across the Spectrum. Chest, 139, 1221-1231. http://dx.doi.org/10.1378/chest.10-0783 